10.2478/aucft-2019-0010

\title{
SCREENING AND IDENTIFICATION OF NEW TYPES OF EXOPOLYSACCHARIDES- PRODUCING LACTIC ACID IN THE INNER MONGOLIA DAIRY PRODUCTS
}

\author{
- Research paper -
}

\author{
Wenfei SHANGGUAN *, He CHEN *,Yilin LI *, Zihua WANG**, Hongxing GUO***, \\ Jiangpeng $\mathrm{MENG}^{1 * * *}$
}

\author{
* School of Food and Biological Engineering, Shaanxi University of Science \& Technology, Xi'an, \\ 710021, China \\ ** College of Food science and Engineering, Ocean University of China, Qingdao,266003, China \\ *** Department of Research and Development, Xi'an Baiyue Goat Milk Corp., Ltd., Xi'an \\ 710089, China
}

\begin{abstract}
Exopolysaccharides (EPS) is a type of polysaccharide produced by lactic acid bacteria (LAB) that can be directly used in foods to make the products more excellent. Therefore, batch studies were performed to explore the effect of different LAB on the production of EPS and antioxidant activity. Five strains with high EPS yield and antioxidant activity were screened out from 66 strains isolated from Tibetan dairy products. The results show that EPS produntion of the five strains (B55, B62, B30, 7830 and K2) were 110.66, 145.48, 132.78, 122.11 and $111.72 \mathrm{mg} \cdot \mathrm{L}^{-1}$, respectively, and they have a higher DPPH free radical scavenging activity $(56.29,66.43,62.94,68.71,61.87 \%)$. Five LAB strains were identified and classified based on screening, purification and 16S rDNA sequences. Molecular characterization based on partial sequence 16S rDNA homology confirmed the initial identification as Lactobacillus fermentum (B55, B62), Lactobacillus plantarum (7830), Pediococcus acidilactici (B30) and Lactobacillus helveticus (K2).
\end{abstract}

Keywords: Exopolysaccharides; lactic acid bacteria; DPPH radical scavenging activity; Phylogenetic tree

\section{INTRODUCTION}

Exopolysaccharides (EPS) is a type of carbohydrate compounds by lactic acid bacteria (LAB) secreted into the culture medium during growth and metabolism. It is the product of microbial adaptation to the environment. EPS are water-soluble glue with unique physical properties. EPS production have received extensive attention as they are produced by food-grade microorganisms with GRAS (generally recognized as safe) and alternatives to the polysaccharides of plant and animal origin. It was reported that LABs isolated from Inner Mongolian traditional yoghurt have the ability to produce EPS (Zhang, 2014) and the EPS produced by LAB has the ability to impart beneficial rheological and functional properties to foods such as yogurt, as a natural thickener, the product has a suitable viscosity and reduced synergy.

Mende et al. (2007) isolated EPS from Streptococcus thermophilus and applied it to yoghurt. It was found that EPS can increase the

Received: 18.09.2019.

Accepted in revised form: 03.12.2019 apparent viscosity of yogurt significantly. The EPS with negatively charged would be attracted electrostatically to casein particles with positively charged in milk, enhancing the polysaccharideprotein network structure. Purwandari et al. (2007) showed that EPS could make yoghurt have better rheological properties and the relationship between its content and yoghurt texture was not obvious. Zhang et al. (2011) added EPS to yoghurt at 1\% inoculum size which produced from Streptococcus thermophilus ST1, that found the viscosity of yoghurt was improved. Girard et al. (2007) thought that the formation of yogurt gel was closely related to the kinetics of strain acidification and the paticipation of EPS can improve the consistency and viscosity of the final obtain yogurt. The study found the some EPS have the ability to antitumoral activites and cholesterol-lowering activity (Ouwehand et al., 2002; Parvez et al., 2006). It is because the EPS produced by LAB would be effected by the probiotic organism and the EPS secreted that the properties of final product would be enhanced obviously.

The important cause of oxidative damage is free radicals which is also important indicators for

\footnotetext{
${ }^{1}$ Corresponding author. E-Mail address: byjpmeng@gmail.com
} 
evaluating the antioxidant properties. Wu et al. ( $\mathrm{Wu}$ et al., 2010) considered that the EPS produced by Bifidobacterium could help prevent gastrointestinal infection and inhibit the growth of harmful bacteria in food. Zhang et al. (2012) studied the in vitro antioxidant activity of EPS which isolated from Lactobacillus plantarum C88 and found that the EPS has the functions of scavenging oxygen free radicals, increasing antioxidant enzyme activity and reducing lipid peroxidation. Uchida et al. (2010) studied the hypocholesterolemic effect of EPS from L. lactis in vivo and found that oral administration of EPS could effectively reduce the incidence of atherosclerosis in hyperlipidemic rabbits and inhibit disease progression. The study on EPS structure of LAB has important significance in theory and application, because the structure and function of LAB EPS are inextricably linked (Yang et al., 2007).

In recent decades, The LAB-EPS development have been vigorously carried out due to the special advantages in product structure, performance and production. The development of new microbial EPS has become one of the hot spots in industrial microbiology research. Quantity production of polysaccharides was authorized in 1947, the yield of dextran from Leuconostoc mesenteroides were 2000 metric tonnes, and FDA approval of food grade xanthan extracted from Xanthomonas campestris in 1969 which was consumed more than 20000 tonnes per year, mainly used as thickener (Sutherland, 2002). Since $L A B$ are food-grade industrial producing bacteria which have a higher safety compared with other bacteria. The research on LAB-EPS has gradually increased in just the past few years. However, the low yield and poor stability of strains are still the dominant factors that restrict their large-scale production. In-depth study of probiotic media to increase extracellular polysaccharide production will provide greater possibilities for the development of the probiotic industry.

The use of traditional polysaccharides is strictly limited, it is because they lack reproducibility in terms of nature, purity, supply and cost. Furthermore, their rheological properties may not fully meeting the requirements. It is finds that novel polysaccharides not only meets most needs of food industry, but also has the ability to be an efficient substitute for the traditional polysaccharides. Therefore, the development of LAB-EPS as a new type of polysaccharide is promising, and $\mathrm{LAB}$ that produce EPS have also become potential strains. The purpose of this experiment is to screen out strains which can be used as a starter in milk that have both EPS-producing and anti-oxidant properties. In this experiment, 66 strains of the laboratory-preserved strains were screened to obtain 5 strains that produced antioxidant EPS and the strains were identified.

\section{MATERIALS AND METHODS}

\section{Microorganism and culture conditions}

The LAB were named previously and isolated from different sources like Kefir grains, commercial dairy product and fermented bovine milk from Inner Mongolia (designated as K1, K2, K3, K4, K5, K6, K7, K8, K9, K10, K11, K12, K13 K14, K15, K16, L49, 7469, 7830, 8014, B25, B26, B30, B43, B55, B56, B62, B82, B90, BB01, GC, GD, L13, L20, L21, L22, L24, L28, L35, L4, L41, L48, L5, L54, L57, L60, L61, L64, L86, La5, LB6, LC, LD, Lp621, Lp69, Lpp, P36, P71, S46, S47, S72, S73, S75, S76, S79 and S91).

The above $66 \mathrm{LAB}$ isolates were maintained in the collection of the School of Food and Biological Engineering, Shaanxi University of Science and Technology. The different strains were each incubated for 24 hours in the MRS (De Man, Rogosa and Sharpe) at an inoculum size of 5\% to obtain the activity. All strains were sub-cultured in their appropriate medium at $37^{\circ} \mathrm{C}$.

\section{Preparation of crude EPS}

The 66 strains of laboratory freeze-dried bacteria powder were activated for three generations in the basic sugar-producing medium. The EPS was prepared in MRS medium by incubating third generation inoculum $\left(10^{9} \mathrm{CFU} \cdot \mathrm{mL}^{-1}\right)$ in static condition for 48 hours at $37^{\circ} \mathrm{C}$. Then to remove the cell pellet, the bacterial suspension was centrifuged at $8000 \mathrm{r} / \mathrm{min}$ for 15 minutes with the teperature of $4{ }^{\circ} \mathrm{C}$ and the supernatant was addedd with $10 \%$ TCA (Trichloroacetic acid solution) to remove protein, then double volume-chilled ethanol was added and the mixture was stored overnight at $4{ }^{\circ} \mathrm{C}$. The mixture was centrifuged at $8000 \mathrm{r} \cdot \mathrm{min}^{-1}$ for 15 minutes and the pellet (crude EPS) collected was dissolved in deionized water (Savadogo et al., 2004). The total amount of carbohydrates in the pellet was estimated using the method of phenolsulphuric acid method as described below.

\section{Rescreening strains producing EPS}

Some strains with the potential to produce more EPS were screened out from 66 strains. The 
operation of rescreening was same as the previous screening. The crude polysaccharide particles dissolved in distilled water and put in a dialysis bag for 24 hours, which operation would remove small molecules such as monosaccharides, pigments, and peptides. The absorbance of polysaccharides was measured by phenol-sulfuric acid method meanwhile the DPPH radical scavenging activity and the ferrous ion chelation rate were measured.

\section{Phenol-Sulphuric Acid Method for EPS detection}

EPS were estimated as total carbohydrates by phenol-sulphuric acid method (Dubois et al., 1956). Glucosidic bond could be hydrolyzed Under the action of concentrated sulfuric acid, these hydrolysed neutral sugars are then partially dehydrated with the elimination of three molecules of water to form furfural or furfural derivatives which could be measured at $490 \mathrm{~nm}$. $1 \mathrm{ml} 5 \%$ $\left(\mathrm{w} \cdot \mathrm{v}^{-1}\right)$ phenol was added to $1 \mathrm{ml}$ sample and $5 \mathrm{ml}$ concentrated sulphuric acid was followed was an ice condition. Then keep the mixture at room temperature for $20 \mathrm{~min}$ and the absorbance value was determined at $490 \mathrm{~nm}$.

Get the standard curve shown in Equation: $y=0.0034 x+0.0134\left(R^{2}=0.9934\right)$.

\section{DPPH radical scavenging activity}

DPPH radical scavenging capacity was determined by the previous method (Wang et al., 2013). $3.0 \mathrm{~mL}$ sample solutions was added in DPPH solution $(0.1$ $\mathrm{mmol} \cdot \mathrm{L}^{-1}$ in $95 \%$ ethanol), then keep the mixture at room temperature in the dark for $30 \mathrm{~min}$ and the absorbance of the mixture was measured at $517 \mathrm{~nm}$. The control group added 95\% ethanol solution without DPPH to the sample, and the blank group used ethanol instead of the sample.

The scavenging activity was calculated using Equation:

$$
\text { Scavenging activity }(\%)=\left[A_{0}-\left(A_{i}-A_{j}\right)\right] \times 100 \% /
$$$$
A_{0} \text {, }
$$

where $\mathrm{A}_{0}$ was the absorbance of the DPPH solution without sample solution, the sample part was replaced by $95 \%$ ethanol, Ai was the absorbance of the sample with DPPH solution and $\mathrm{A}_{j}$ was the absorbance of the sample with the same condition as $\mathrm{A}_{i}$, but with $95 \%$ ethanol added to replace DPPH solution.

\section{The ferrous ion chelation rate}

The ferrous ion chelating rate was assessed according to the method reported by Ma et al., 2013, with minor modifications. One millilitre of samples at different concentrations was mixed with $0.1 \mathrm{~mL}$ $\mathrm{FeCl}_{2}\left(2 \mathrm{mmol} \cdot \mathrm{L}^{-1}\right), 0.2 \mathrm{~mL}$ ferrozine $\left(5 \mathrm{mmol} \cdot \mathrm{L}^{-1}\right)$ and $3.7 \mathrm{~mL}$ distilled water were added to the mixture, shaken well, then incubated for $20 \mathrm{~min}$ at room temperature. The absorbance of the supernatant was determined at $562 \mathrm{~nm}$. Replace the sample with water was used as the positive standard. The ferrous ion chelating activity was calculated by the following Equation:

Ferrous ion chelating rate $(\%)=\left(A_{2}-A_{1}\right) \times 100 \% / A_{2}$ where $A_{1}$ was the absorbance of the sample solution and $A_{2}$ was the absorbance of the positive standard same condition as $\mathrm{A}_{1}$.

\section{DNA extraction and amplification of 16S rDNA sequences}

Molecular methods are powerful and efficient tools to classify bacteria taxonomically even between closely related species. These methods are mostly based on polymerase chain reaction (PCR). Genotypic characterization of microorganisms according to their $16 \mathrm{~S}$ ribosomal RNA sequencing is the best and most reliable approach to identify bacteria on a phylogenetic basis.

The use of molecular tools has broken through the limitations of traditional methods in the identification of bacteria. These techniques are based on PCR using oligonucleotide primers to amplify targeted DNA fragments. These PCR primers are designed at different levels of classification, from genus-specific (Schillinger et al., 2008), to the species-specific level (Coton et al., 2008), to sub-species level ( $\mathrm{Pu}$ et al., 2002), and also further to the strain-level (Coudeyras et al., 2008). This method is suitable for the initial identification of bacterial species by other methods (usually phenotypic methods).

A pure colony which was picked from each probiotic strain was picked was lysed in $50 \mathrm{~mL}$ TaKaRa Lysis Buffer (Cat. No. 9164) (Goranov et al., 2015). After 15 min denaturation at $80{ }^{\circ} \mathrm{C}$, then via centrifugation $(14,000 \mathrm{~g})$ the supernatant was used as a template. A TaKaRa 16S rDNA Bacterial Identification PCR Kit (Cat. No. RR176) was using to perform the amplification of the target fragment. The target fragment was amplified by polymerase chain reaction (PCR) using 'Forward primer/ Reverse primer2' and could be used for amplification of full sequence DNA fragments. The reaction system and reaction conditions are described as Table 1 shows.

\section{Purification of PCR products}

According to the instruction, a Takara MiniBEST Agarose Gel DNA Extraction Kit Ver.4.0 (Cat. No. 9762) was used to purified the amplified 16S rDNA fragments and the electrophoresis used DL 2,000 DNA Maker which including 2000, 1000, 750, 500, 250 and $100 \mathrm{bp}$ fragments. 
DNA sequencing and construction of phylogenetic tree

The 16S rDNA gene fragments sequencing was performed by ABI PRISMTM 33730XL DNA Sequence (Applied Biosystem) by using Primer of Seq forward, Seq reverse and Seq internal primers (Table 2). In oder to construct phylogenetic trees, the sequences were analyzed by the NCBI (National Center of Biotechnology Information) BLAST (basic local alignment search tool) and the neighbor-joinong method was used to infer evolutionary history with the unweighted pair group method with arithmetic-mean (UPGMA) (Sokal, 1958). The evolutionary history of the taxa analyzed was represented by taking bootstrap consensus tree inferred from 1000 replicates and the maximum composite likelihood method was used to compute the evolutionary distances.

Table 1. PCR reaction conditions

\begin{tabular}{|c|c|c|c|c|}
\hline Reaction system & \multicolumn{4}{|c|}{ Reaction conditions } \\
\hline Supernatant (after denaturation) & $1 \mu \mathrm{L}$ & $94^{\circ} \mathrm{C}$ & $5 \mathrm{~min}$ & 1 cycle \\
\hline PCR premix & $25 \mu \mathrm{L}$ & $94^{\circ} \mathrm{C}$ & $1 \mathrm{~min}$ & \\
\hline Forward primer $(20 \mathrm{pmol} / \mathrm{mL})$ & $0.5 \mu \mathrm{L}$ & $55^{\circ} \mathrm{C}$ & $1 \mathrm{~min}$ & 30 cycles \\
\hline Reverse primer2 $(20 \mathrm{pmol} / \mathrm{mL})$ & $0.5 \mu \mathrm{L}$ & $72{ }^{\circ} \mathrm{C}$ & $1.5 \mathrm{~min}$ & \\
\hline $16 \mathrm{~S}$-free $\mathrm{H}_{2} \mathrm{O}$ & $23 \mu \mathrm{L}$ & $72{ }^{\circ} \mathrm{C}$ & $5 \mathrm{~min}$ & 1 cycle \\
\hline Total volume & $50 \mu \mathrm{L}$ & & & \\
\hline
\end{tabular}

Table 2. Sequencing primer

\begin{tabular}{ll}
\hline Primer & Primer sequence \\
\hline Seq forward & 5'-GAGCGGATAACAATTTCACACAGG-3' \\
Seq reverse & 5'-CGCCAGGGTTTTCCCAGTCACGAC-3' \\
Seq internal & 5'-CAGCAGCCGCGGTAATAC-3' \\
\hline
\end{tabular}

\section{RESULTS AND DISCUSSIONS}

\section{Potential strains were screened out by measuring EPS production}

Table 3 shows that 11 strains (Lp69, LB6, L13, L64, 8014, 7830, K2, B62, B55, B30, S72) were screened out with potential for EPS production compared with other strains, their EPS yield were 134.94, $172.82,163.88,133.51,132.44,131.72,149.94$, $237.15, \quad 187.88, \quad 175.97, \quad 130.24 \quad \mathrm{mg} \cdot \mathrm{L}^{-1}$, respectively. These 11 strains were re-screened to screen out the strains with high-yield EPS with antioxidant activity. The higher content of crude EPS at this time was due to the total carbohydrate content measured by the phenol-sulfuric acid method, while the monosaccharide in the EPS sample wast removed during the initial screening.

\section{Antioxidative EPS-producing strains obtained by rescreening}

The EPS yield, DPPH free radical scavenging rate and ferrous ion chelation rate of 11 strains were determined as shown in Figure 1,. All tested probiotic strains showed the potential to produce EPS and antioxidants. The polysaccharide production of the five strains (B55, B62, B30, 7830 and $\mathrm{K} 2$ ) exceeded $100 \mathrm{mg} \cdot \mathrm{L}^{-1}$ among all the strains these yield of EPS were 110.66, 145.48, 132.78,
122.11 and $111.72 \mathrm{mg} \cdot \mathrm{L}^{-1}$, respectively. This is a new discovery that $\mathrm{K} 2$ was isolated from natural kefir grains among them and the research and analysis of this strain could continue. Their EPS yields were lower than that of the initial screening that because residual monosaccharides in many culture media were separated out after the dialysis. At the same time, they have relatively high DPPH radical scavenging activity $(56.29,66.43,62.94$, $68.71,61.87 \%$ ) and ferrous ion chelating rate. The different performance of various strains when producing EPS were closely related to their inherent factors.

These strains can all be used as potential strains for the production of EPS. Many studies have used LAB-EPS in products to obtain better product. Yang et al. (Yang et al., 2013) extracted the EPS of a Streptococcus thermophilus S1 and added it to buffalo yoghurt in different proportions. The results showed that both the hardness and the viscosity of the yoghurt reached a maximum value when the added amount was $0.01 \%$ and the above two indicators showed a downward trend with the continued increase of EPS added, but they were all greater than the yogurt samples without EPS. The analysis indicates that this phenomenon may be related to the network structure of EPS. 
Table 3. Screening for EPS-producing strains of LAB

\begin{tabular}{lllllllll}
\hline No. & LAB & EPS $\left(\mathrm{mg} \cdot \mathrm{L}^{-1}\right)$ & No. & LAB & EPS $\left(\mathrm{mg} \cdot \mathrm{L}^{-1}\right)$ & No. & LAB & EPS $\left(\mathrm{mg} \cdot \mathrm{L}^{-1}\right)$ \\
\hline 1 & L49 & $74.54 \pm 1.1$ & 23 & LD & $21.19 \pm 1.58$ & 45 & Lpp & $19.5 \pm 0.98$ \\
2 & Lp69 & $134.93 \pm 1.2$ & 24 & 7469 & $23.87 \pm 1.52$ & 46 & S76 & $27.44 \pm 1.17$ \\
3 & L28 & $56.67 \pm 1.34$ & 25 & L22 & $17.16 \pm 0.83$ & 47 & L86 & $20.08 \pm 0.66$ \\
4 & L41 & $76.33 \pm 1.25$ & 26 & LP621 & $25.88 \pm 1.42$ & 48 & GC & $40.67 \pm 2.10$ \\
5 & LB6 & $172.81 \pm 1.68$ & 27 & K1 & $81.54 \pm 1.72$ & 49 & S72 & $130.23 \pm 1.36$ \\
6 & L5 & $75.25 \pm 2.3$ & 28 & K2 & $149.94 \pm 1.58$ & 50 & GD & $22.88 \pm 1.35$ \\
7 & L48 & $70.97 \pm 1.34$ & 29 & K5 & $81.54 \pm 0.82$ & 51 & S75 & $22 \pm 1.24$ \\
8 & L54 & $71.68 \pm 1.96$ & 30 & K11 & $11.80 \pm 1.47$ & 52 & S79 & $15.52 \pm 0.84$ \\
9 & La5 & $78.83 \pm 1.54$ & 31 & K15 & $33.26 \pm 0.67$ & 53 & S91 & $31.11 \pm 1.37$ \\
10 & L60 & $74.18 \pm 1.63$ & 32 & L20 & $18.50 \pm 0.74$ & 54 & P71 & $13.47 \pm 1.32$ \\
11 & L13 & $163.88 \pm 1.53$ & 33 & B56 & $118.76 \pm 1.39$ & 55 & P36 & $57.58 \pm 1.25$ \\
12 & L64 & $133.50 \pm 1.48$ & 34 & B43 & $90.23 \pm 0.65$ & 56 & K4 & $98.61 \pm 0.75$ \\
13 & LC & $81.69 \pm 1.42$ & 35 & B62 & $237.14 \pm 0.59$ & 57 & K3 & $117.73 \pm 0.86$ \\
14 & L57 & $65.25 \pm 0.75$ & 36 & B90 & $119.5 \pm 0.77$ & 58 & K12 & $20.23 \pm 1.11$ \\
15 & L21 & $76.33 \pm 1.72$ & 37 & B25 & $114.94 \pm 0.92$ & 59 & K16 & $28.61 \pm 1.15$ \\
16 & 8014 & $132.43 \pm 1.32$ & 38 & B55 & $187.88 \pm 1.65$ & 60 & K13 & $19.5 \pm 1.24$ \\
17 & 7830 & $131.72 \pm 1.76$ & 39 & B82 & $94.35 \pm 0.59$ & 61 & K6 & $21.41 \pm 0.58$ \\
18 & L4 & $78.47 \pm 0.98$ & 40 & B30 & $175.97 \pm 1.36$ & 62 & K7 & $105.67 \pm 0.99$ \\
19 & L61 & $72.39 \pm 1.36$ & 41 & B26 & $107.58 \pm 1.45$ & 63 & K8 & $117.88 \pm 0.62$ \\
20 & L24 & $53.81 \pm 0.85$ & 42 & S46 & $97.14 \pm 1.28$ & 64 & K9 & $108.61 \pm 0.59$ \\
21 & L35 & $15.82 \pm 1.48$ & 43 & S47 & $117.58 \pm 1.53$ & 65 & K10 & $42 \pm 1.37$ \\
22 & BB01 & $86.90 \pm 0.86$ & 44 & S73 & $90.38 \pm 1.49$ & 66 & K14 & $43.76 \pm 1.42$ \\
\hline
\end{tabular}

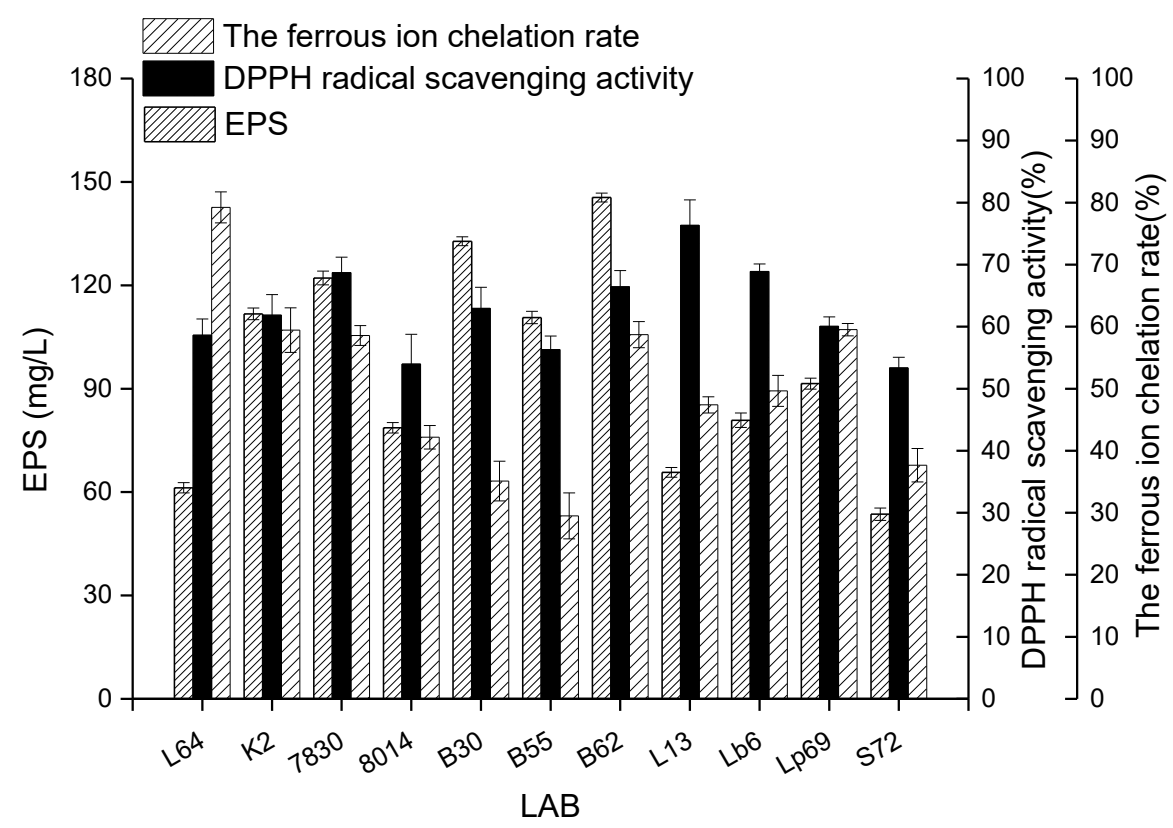

Figure 1. EPS production and antioxidant activity of the selected $11 \mathrm{LAB}$

Prasanna et al. (2012) screened 4 EPS-producing strains from 22 Bifidobacterium strains. The correlation analysis of the apparent viscosity and $\mathrm{pH}$ of fermented milk showed that the EPS produced by these four strains helps increase the viscosity of dairy products during milk acidification.

A large number of studies have shown that some LAB-EPS have antioxidant effects. Xu et al. (2012) studied the antioxidant activity of EPS produced by 
L. casei KW3. It showed that when the EPS concentration was $200 \mu \mathrm{g} \cdot \mathrm{mL}^{-1}$, the DPPH radical scavenging rate was $37.53 \%$ In vitro experiments. Meng et al. (2012) found that the antioxidant activity of EPS from Lactobacillus paracasei $\mathrm{H} 9$ increased with increasing EPS concentrations. The DPPH radicals rates was $(70.8 \pm 0.6) \%$ when the EPS concentration reached $200 \mathrm{mg} \cdot \mathrm{L}^{-1}$. The abovementioned studies on the yield of EPS and antioxidation performance obtained were much than this experiment, which mainly because this experiment did not optimize the culture medium. At the end of the experiment, the culture medium will be optimized to obtain more EPS production and antioxidant activity.

\section{Phylogenetic analysis by 16S rDNA analysis}

The results of the agarose gel electrophoresis (Figure 2) showed that after sequencing, the length of all amplicons exceeded expectations, reaching 1000 bp, 1394 bp (B55), 1396 bp (B30), 1410 bp (7830), $1431 \mathrm{bp}(\mathrm{K} 2)$ and $1358 \mathrm{bp}$ (B62). After amplification and purification of the target fragment, the five strains was preliminary identified as L. fermentum (B55, B62), L. plantarum (7830), $P$. acidilactici (B30) and L. helveticus (K2) via molecular characterization based on 16S rDNA homology of partial sequences (B55, B62, B30, 7830 and K2) with the sequences available in the NCBI database. In order to track the evolutionary patterns of isolates based on sequence similarity, a phylogenetic tree was constructed using the UPGMA adjacent ligation method between the 16S rDNA sequence of the strain in the NCBI database and the corresponding sequence of different probiotic lactic acid bacteria (Sun et al., 2016). The resulting trees of Figure 3 showed that strain B55 had 100\% homology with L. fermentum NCDO $1750,63 \%$ homology with $L$. fermentum CIP 102980 and L. fermentum NBRC 15885. B62 had the $100 \%$ homology relationship with $L$. fermentum strain CIP 102980 and L. fermentum strain NBRC 15885. Similarly, $80 \%$ homology with $P$. acidilactici strain DSM 20284 was shown in the tree of B30 and both had $99 \%$ homology with $P$. acidilactici strain NGRI 0510Q, 99\% homology was found for strain 7830 with L. plantarum strain JCM 1149, L. plantarum strain NBRC 15891, $L$. plantarum strain CIP 103151 and L. plantarum strain NRRL B-14768, strain K2 had $100 \%$ homology with $L$. helveticus strain NBRC 15019 and L. helveticus strain DSM 20075. Overall, due to the high production of EPS potential, the five strains was selected for further study as L. fermentum (B55, B62), P. acidilactici (B30) L. helveticus (K2) and $L$. plantarum (7830) via molecular phylogenetic analysis.

Wang et al. (2015) found that Lactobacillus plantarum $\mathrm{YW} 11$ can produce $90 \mathrm{mg} \cdot \mathrm{L}-1$ of EPS in semi-custom medium, which was isolated from Tibet Kefir. The results showed lower EPS yield than 7830 in this study, mainly because of differences in species and culture media.

The production, characterization and functional properties in vitro of EPS of Lactobacillus helveticus MB2-1 with whey solution as basal medium were investigated by Li et al. (2014) It is repoted that in the case of lactose and soy protein ept $80 \mathrm{~g} \cdot \mathrm{L}-1$ and $20 \mathrm{~g} \cdot \mathrm{L}-1$, respectively, the maximum yield of EPS was $753 \mathrm{mg} \cdot \mathrm{L}-1$. EPS also exhibits effective antioxidant effects such as the ability to scavenge hydroxyl groups, superoxide and DPPH free radicals, and the ability to chelate ferrous ions (related to dose). Since the fermentation conditions have not yet been optimized and the strains were not the same in this study, the amount of EPS produced by the Lactobacillus helveticus MB2-1 were higher than K2 (isolated from Kefir in this experiment). At the same time, $\mathrm{Li}$ et al. also confirmed that the polysaccharides have better antioxidant properties.

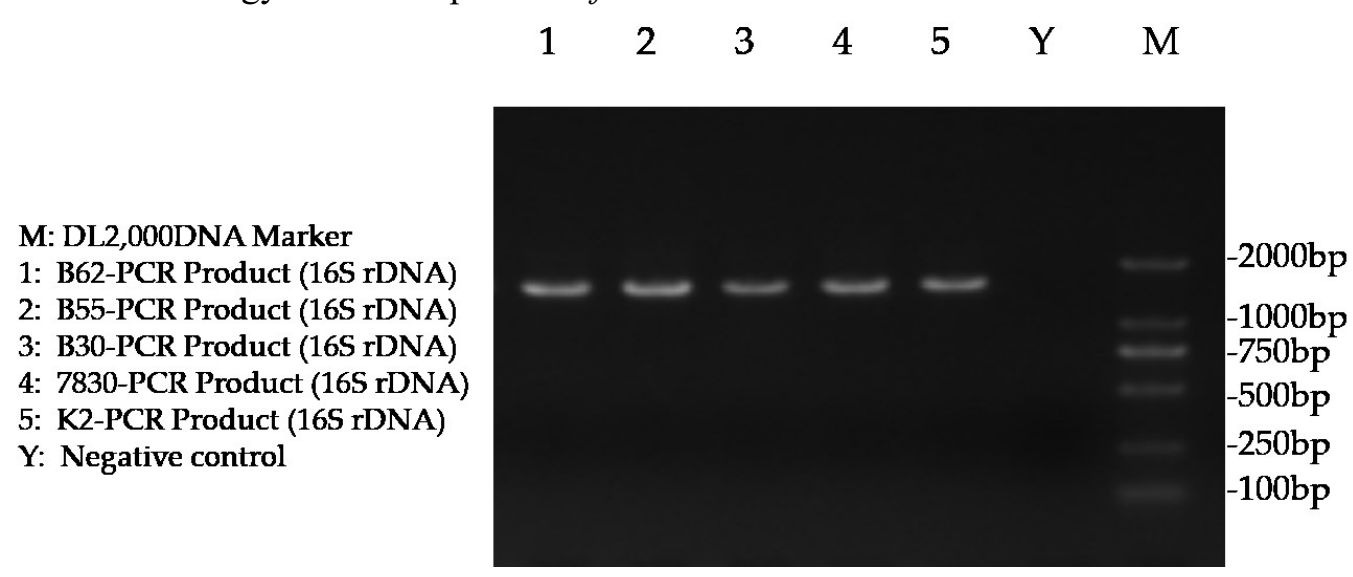

Figure 2. Agarose gel electrophoresis of PCR products from genomic DNA isolated from representative LAB strains. 

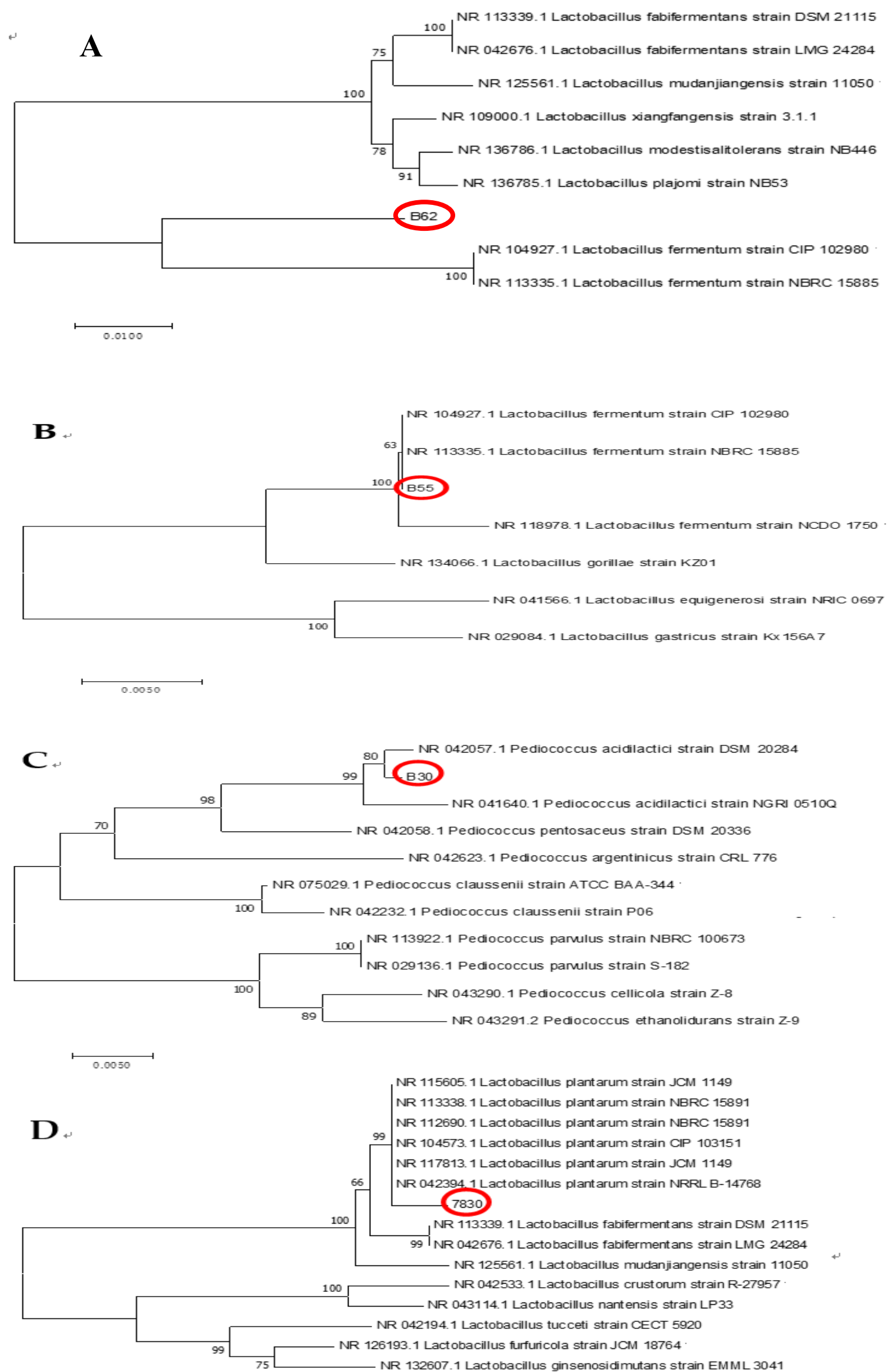

$\stackrel{\longmapsto}{0.0050}$ 


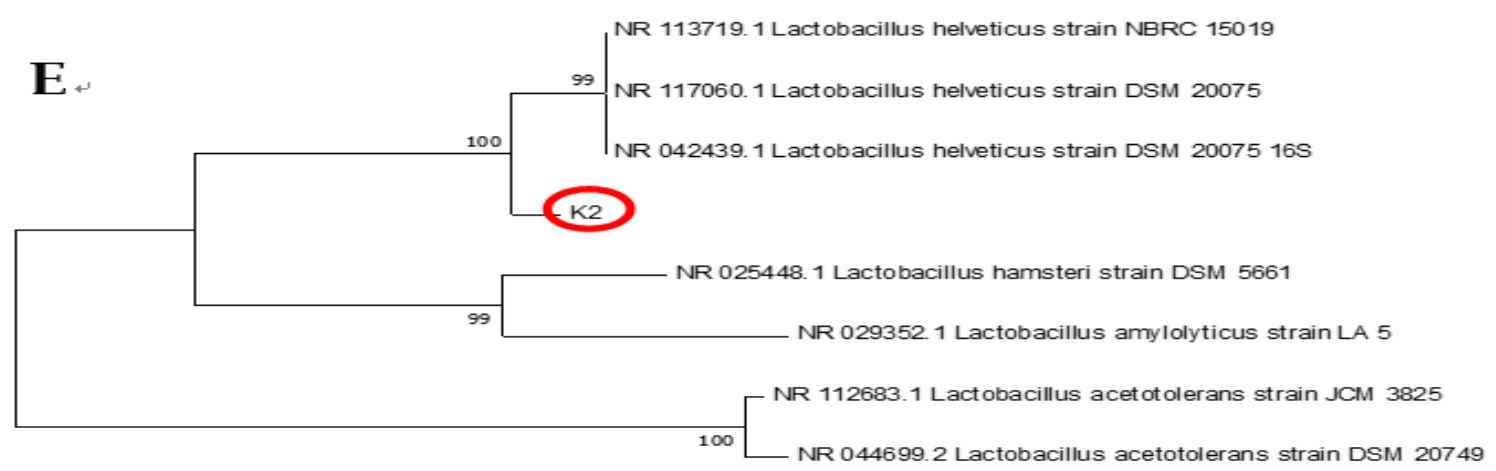

$\stackrel{\longmapsto .0050}{\longmapsto}$

Figure 3. Phylogenetic tree of concatenated LAB strain B62 (A), B55 (B), B30 (C) 7830 (D) and K2 (E) constructed by the Neighbour-joining method with Kimura 2-parameter as evolution model and based on 16S rDNA nucleotide sequences.

Fukuda et al. (2010) showed that a thermophilic LAB, Lactobacillus fermentum TDS030603, produced about $100 \mathrm{mg} \cdot \mathrm{L}^{-1}$ of EPS in purified form when grew in MRS broth. Zhang et al. (2011) found a Lactobacillus fermentum F6 present in traditional dairy products in Inner Mongolia, China with an EPS yield of $20 \mathrm{mg} \cdot \mathrm{L}-1.1 \%(\mathrm{w} / \mathrm{v})$ EPS aqueous solution exhibits higher viscosity, indicating that the EPS has potential as a food tackifier. The yields of the two strains (B62 and B55) of Lactobacillus fermentum which have the potential to produce polysaccharides in this study were all higher than the above studies.
Strain M76 was selected as an EPS-producing functional strain for the ability of DPPH free radicals to have antioxidant activity in makgeolli, a Korean fermented rice wine by Song et al. (Song et al., 2013). Strain M76 has a high identity $(99.0 \%)$ with $P$. acidilactici according to the 16S rRNA gene sequencing analysis. And the soluble EPS produced by $\mathrm{M} 76$ can up to $200 \mathrm{mg} \cdot \mathrm{L}^{-1}$, which EPS yield was higher than B30 in this study. Different strains have different EPS production, which is related to the strain itself. Identify the structure of the EPS and find out the relationship between yield and structure in the subsequent study.

\section{CONCLUSIONS}

The results of this study indicated that all 66 tested strains had some ability to produce EPS. Among the 66 strains, B62 had the highest EPS yield of 145.48 $\mathrm{mg} / \mathrm{L}$ and had the strongest function of eliminating DPPH free radicals $(66.43 \%)$. In addition, the EPS yields of B55, B30, 7830 and $\mathrm{K} 2$ were all greater than $100 \mathrm{mg} \cdot \mathrm{L}-1$, ie $10.66,132.78,122.11$ and $111.72 \mathrm{mg} \cdot \mathrm{L}-1$, respectively. They also had the antioxidant function of eliminating DPPH free radicals, respectively 56.29, 62.94, 68.71 and $61.87 \%$. Basing in $16 \mathrm{~S}$ rDNA homology of partial sequences, the molecular characterization were confirmed the preliminary identification as L. fermentum (B55, B62), L. plantarum (7830), P. acidilactici (B30) and L. helveticus (K2). These five LAB strains can be used as potential strains for the production of EPS which also can be applied to the products after optimized culture conditions and purification.

\section{ACKNOWLEDGEMENTS}

The work was partially supported by the Key Research and Development Program of Shaanxi (Program No. 2019ZDLNY06-01)., the science and technology project of Xi'an city [201806118YF06NC14(2)] and the Science and Technology Bureau of Weiyang District (No.201831).

\section{REFERENCES}

1. Coton, M.; Berthier, F.; Coton, E. (2008). Rapid identification of the three major species of dairy obligate heterofermenters Lactobacillus brevis, Lactobacillus fermentum \& Lactobacillus parabuchneri by species-specific duplex PCR. Fems Microbiol Lett. 2008, 284, 150-157.DOI: 10.1111/j.15746968.2008.01206.x. 
2. Coudeyras, S.; March\&in, H.; Fajon, C.; Forestier, C. (2008). Taxonomic \& strain-specific identification of the probiotic strain Lactobacillus rhamnosus 35 within the Lactobacillus casei group. Appl Environ Microbiol. 2008, 74, 2679-2689. DOI: 10.1128/aem.02286-07.

3. Dubois, M.; Gilles, K.; Hamilton, J.; Rebers, P.; Smith, F. (1956). Colorimetric methods for determination of sugars and related substances. Analytical Chemistry. 1956, 28, 350-356. DOI: 10.1021/ac60111a017.

4. Fukuda, K.; Shi, T.; Nagami, K. (2010). Effects of carbohydrate source on physicochemical properties of the exopolysaccharide produced by Lactobacillus fermentum, TDS030603 in a chemically defined medium. Carbohydrate Polymers. 2010, 79(4), 1040-1045. DOI: 10.1016/j.carbpol.2009.10.037.

5. Girard, M.; Sehaffer-Lequart, C.(2007). Attractive in ieractions between selected anionic exopolysaecharides and milk proteins. Food Hydroeolloids. 2007, 9, 1-10. DOI: 10.1016/j.foodhyd.2007.09.001.

6. Goranov, B.; Denkova, R.; Teneva, D. (2015). Molecular-genetic identification of Lactobacillus strains, isolated from homemade yoghurt. Ukrainian Food J. 2015, 4(1), 67-76.

7. Li, W.; Ji, J.; Rui, X. (2014). Production of exopolysaccharides by Lactobacillus helveticus, MB2-1 and its functional characteristics invitro. LWT - Food Science and Technology. 2014, 59(2), 732-739. DOI: 10.1016/j.lwt.2014.06.063.

8. Mende, S.; Peter, M.; Bartels, K.; et al.(2013) Addition of purified exopolysaccharide isolates from $S$. thermophilus to milk and their impact on the rheology of acid gels. Food Hydroeolloids, 2013, 32, 178185. DOI: 10.1016/j.foodhyd.2012.12.011.

9. Meng, X.S.; Li, B. (2012). Antioxidant activity of Lactobacillus paracase and its exopolysaccharides. Food Sci Tech, 2012, 37(09), 48-53. DOI: 10.13684/j.cnki.spkj.2012.09.001.

10. Ouwehand, A.; Salminen, S.; Isolauri, E.(2002). Probiotics: an overview of beneficial effects. Antonie van Leeuwenhoek. 2002, 82, 279-289. DOI: 10.1023/a:1020620607611.

11. Parvez, S.; Malik, K.A.; Kang, S.A.; Kim, H.Y.(2006). Probiotics and their fermented food products are beneficial for health. $J$ Appl Microbiol. 2006, 100, 1171-1185. DOI: 10.1111/j.1365-2672.2006.02963.x.

12. PRASANNA, P.H.P.; GRANDISON, A.S.; CHARALAMPOPOULOS, D. (2013). Screening human intestinal Bifidobacterium strains for growth, acidification, EPS production and viscosity potential in low fat milk. Int Dairy J, 2012, 23(1), 36-44. DOI: 10.1016/j.idairyj.2011.09.008.

13. Pu, Z.Y.; Dobos, M.; Limsowtin, G. K. Y.; Powell, I. B. (2002). Integrated polymerase chain reactionbased procedures for the detection and identification of species and subspecies of the Gram-positive bacterial genus Lactococcus. $J$ Appl Microbiol. 2002, 93, 353-361.DOI: 10.1046/j.13652672.2002.01688.x.

14. Purwandari, U.; Shah, N.P.; Vasiljevic, T.(2007). Effects of exopolysaccharide-producing strains of Streptococcus thermophilus on technological and rheological properties of set-type yoghurt. Int Dairy J, 2007, 17, 1344-1352. DOI: 10.1016/j.idairyj.2007.01.018.

15. Savadogo, A.C.A.T.; Ouattara1, Savadogo, P.W.; Barro, N.; Ouattara, A.S.; Traoré, A.S.(2004). Identification of exopolysaccharides-producing lactic acid bacteria from Burkina Faso fermented milk samples. Afr J Biotechnol. 2004, 3, 189-194. DOI: 10.5897/AJB2004.000-2034.

16. Schillinger, U.; Boehringer, B.; Wallbaum, S.; Caroline, L.; Gonfa, A.; Huch, M.; Holzapfel, W. H.; Franz, C. (2008). A genus-specific PCR method for differentiation between Leuconostoc and Weissella and its application in identification of heterofermentative lactic acid bacteria from coffee fermentation. Fems Microbio Lett. 2008, 286, 222-226. DOI: 10.1111/j.1574-6968.2008.01286.x.

17. Sokal, R.R. (1958). A statistical method of evaluating systematic relationships. Univ Kansas Sci Bull. $1958,38,1409-1438$.

18. Song, Y.R.; Jeong, D.Y.; Cha, Y.S. (2013). Exopolysaccharide produced by Pediococcus acidilactici M76 isolated from the Korean traditional rice wine, makgeolli. J Microbiol Biotechnol. 2013, 23(5), 681-688. DOI: $10.4014 / \mathrm{jmb} .1301 .01032$.

19. Sun, G.; Yao, T.; Feng, C. (2017). Identification and biocontrol potential of antagonistic bacteria strains against Sclerotinia sclerotiorum, and their growth-promoting effects on Brassicanapus. Biol Control. 2017, 104, 35-43. DOI: 10.1016/j.biocontrol.2016.10.008.

20. Sutherland, I.W.(2002). A sticky business. Microbial exopolysaccharides: current products and future trends. Microbiology Today. 2002, 29, 70-71.

21. Uchida, M.; Ishii, I.; Inoue, C.(2010). Kefiran reduces atherosclerosis in rabbits fed a high cholesterol diet. J Atheroscler Thromb, 2010, 17, 980-988. DOI: 10.5551/jat.4812.

22. Wang, J.; Zhao, X.; Tian, Z. (2015). Characterization of an exopolysaccharide produced by Lactobacillus plantarum YW11 isolated from Tibet Kefir. Carbohyd Polym. 2015, 125, 16-25. DOI: 
10.1016/j.carbpol.2015.03.003

23. Wang, Q.; Sun, Y.; Yang, B.; Wang, Z.; Liu, Y.; Cao, Q.; Sun, X.; Kuang, H. (2014). Optimization of polysaccharides extraction from seeds of Pharbitis nil and itsanti-oxidant activity. Carbohyd. Polym. 2014, 102, 460-466. DOI: 10.1016/j.carbpol.2013.11.068.

24. Wu, M.H.; Pan, T.M.; Wu, J.Y.(2010). Exopolysaccharide activities from probiotic bifidobacterium: Immunomodulatory effects (on J774A.1 macrophages) and antimicrobial properties. Int J Food Microbiol. 2010, 144, 104-110. DOI: 10.1016/j.ijfoodmicro.2010.09.003.

25. Xu, N.; Xi, A.D. (2012). Study on exopolysaccharide of Lactobacillus casei KW3. J Chine Inst Food Sci Tech, 2012, 12(7), 42-47. DOI: 10.3969/j.issn.1009-7848.2012.07.007.

26. Yang, T.; Wu, K.; Wang, F. (2014). Effect of exopolysaccharides from lactic acid bacteria on the texture and microstructure of buffalo yoghurt. Int Dairy $J, 2014,34(2)$, 252-256. DOI: 10.1016/j.idairyj.2013.08.007.

27. Yang, Z.N.; Zhang, X.(2007). Rheological characterization and molecular structural modification of exopolysaccharides produced by lactic acid bacteria. Food Sci. 2007, 28(12), 535-538. DOI: CNKI:SUN:SPKX.0.2007-12-132.

28. Zhang, L.; Liu, C.Y.; Li, D.(2013). Antioxidant activity of an exopolysaccharide isolated from Lactobacillus plantarum C88. Int $J$ Biol Macromol, 2013, 54, 270-275. DOI: 10.1016/j.ijbiomac.2012.12.037.

29. Zhang, T.H.; Zhang, C.H.; Li, S.H.(2011). Growth and exopolysaccharide production by Streptococcus thermophilus ST1 in skim milk. Braz J Microbiol, 2011, 42, 1470-1478. DOI: 10.1590/S151783822011000400033.

30. Zhang, Y.C.; Li, S.Y.; Zhang, C.Y.(2011). Growth and exopolysaccharide production by Lactobacillus fermentum F6 in skim milk. Afr J Biotechnol, 2011, 10(11), 2080-2091. DOI: 10.1093/cid/cir036.

31. Zhang, C.L.(2014). Selection of exopolysaccharide-producing lactic acid bacteria isolates from Inner Mongolian traditional yoghurt. Mljekarstvo, 2014 64(4):254-260. DOI: 10.15567/mljekarstvo.2014.0404. 